\title{
Index for Volume 21 of Molecular Plant-Microbe Interactions
}

\section{AUTHOR AND SUBJECT INDEX}

Abe, M., 1175

Acidovorax avenae, on rice, flagellin perception, flg22 receptor, 1635

Actinobacteria, endophytic, Arabidopsis

thaliana, defense pathways, 208

Adams-Phillips, L., 646

Adham, S. A. I., 979

Afzal, A. J., 507

Agrobacterium spp.

$-A$. rhizogenes, rolC gene, oxygen and stress tolerance, 1561

-A. tumefaciens, host infiltration, responses, 1528

Aguilar, O. M., 459, 1232

Ah-Fong, A. M. V., 433

Akimitsu, K., 1591

Akimoto-Tomiyama, C., 361

Albugo candida, on Arabidopsis thaliana:

- and Brassica juncea, immunity, 745

—races and plant resistance, protein, 757

Aldea, M., 1297

Aldwinckle, H. S., 448

Alfalfa (see also Medicago spp.): Sinorhizobium meliloti, symbiosis and tagged mutants, 219

Alkan, N., 1058

Allègre, M., 232

Allocasuarina verticillata, gene silencing, host transcriptional, 518

Alternaria alternata, on tangerine, toxin biosynthesis gene, RNA silencing, 1591

Ambrose, B., 188

Ameline-Torregrosa, C., 61

Aminin, D. L., 1561

Anderson, A. J., 1067

Andreasson, E., 1275

Andrews, D. L., 110

Antibiotic, diacetylphloroglucinol,

Pseudomonas fluorescens source, 1349

Aphid, genomics and plant interaction studies, review, 701

Apoptosis, Leptosphaeria maculans on Brassica napus, lesion formation, 1143

Apple, scab, receptor-like genes and resistance, 448

Arabidopsis: cyst nematode, RNAs and targets, 1622; elicitor treatment, phosphoproteins,

1275; herbivorous insects, resistance induced by microbes, 919; mutant ssi4, dwarfism, 40; Piriformospora indica, auxin interaction, 1371; Piriformospora indica on, drought stress genes, 799; Pseudomonas syringae, water-stressed plants and virulence, 361; Ralstonia solanacearum, transposon mutagenesis, 1261; resistance, signaling pathways, 1285; soybean rust, nonhost resistance, 1421; Turnip mosaic virus, veinal necrosis and HR-like cell death, 260; victorin sensitivity, LOV1 gene, characterization, 7; Xanthomonas campestris, type III secretion regulators, 555

-A. thaliana: actinobacteria, endophytic in defense pathways, 208; Albugo candida on, Brassica juncea compatibility, 745; Albugo candida, races, resistance protein, 757; Bacillus subtilis, salt tolerance, 737; Bradyrhizobium and Pseudomonas spp. on, transcriptome changes, 244; Cucumber mosaic virus, resistance gene RCY1, 1398; cyst nematode, salicylic acid, 424; drought tolerance, bacterial volatile role, 1067; Erwinia amylovora, type three-secreted proteins, cell death and defenses, 1076; Pseudomonas syringae, defense pathway elements, $R$-avr interactions, 646; virus symptoms, quantitative trait loci, 198

ARCHIPELAGO, rice disease resistance, regulation, review, 869

Arnould, C., 232

Arrese-Igor, C., 622

Asopirillum brasilense, on wheat, nitrite reductase gene, 831

Asselbergh, B., 709

Attaran, E., 1482

Auguy, F., 518

Autran, D., 518

Aux, G., 433

Auxin, Arabidopsis and Piriformospora indica interaction, cytokinins, 1371

Avadhani, M., 404

Avrova, A. O., 433

Azevedo, R. A., 891

Azospirillum brasilense, on tomato, nitric oxide production, root effect, 1001

Bacillus subtilis, on Arabidopsis thaliana, salt tolerance, tissue-specific regulation, 737

Bacteria, plant pathogens, virulence attenuated, signaling factors, 326

Bahlawane, C., 1498

Bai, Y., 30

Baillieul, F., 232

Bakker, E. G., 7

Bakker, J., 791

Bakker, P. A. H. M., 1609

Baldwin, I. T., 988

Ballini, E., 859, 869

Bamunusinghe, D., 1106

Barakat, R., 1443

Barash, I., 1094

Barau, J. G., 891

Barker, D. G., 535

Barker, S. J., 1143

Barley, Soilborne cereal mosaic and Soilborne wheat mosaic viruses, resistance responses, 1193

Barny, M.-A., 1076

Barraza, A., 958

Batut, J., 1232

Baudouin, E., 781

Bauer, W. D., 1184

Baum, T. J., 424, 1622

Baurès, I., 1154

Bean pod mottle virus, on soybean, gene silencing, cover photo, May

Becker, A., 219, 947, 1498

Beer, S. V., 1387

Behr, M., 1325

Beker, M. P., 459

Bendahmane, A., 1154

Bender, C. L., 383

Benitez, M., 631, 1297

Bent, A. F., 646, 1285

Bhat, S., 1539

Bhattarai, K. K., 1205
ERRATA PAGE NUMBERS IN ITALICS

Biehl, B. S., 1549

Bilgin, D. D., 1297

Bipolaris sorokiniana, on wheat, resistance conferred by bean PGIP, 171

Birch, P. R. J., 433, 1460

Bishnoi, U., 1205

Bishop, J. G., 820

Blanco, F. A., 459

Blechl, A. E., 171

Bodenhausen, N., 919

Bogusz, D., 518

Bolton, M. D., 1515

Borejsza-Wysocka, E., 448

Borhan, M. H., 757

Borisov, A., 1600

Botrytis cinerea

- conidial growth and germination, signaling pathway, 1443

-NADPH oxidases, pathogenicity, 808

Brader, G., 967

Bradyrhizobium sp., on Arabidopsis thaliana, Pseudomonas syringae interaction, transcriptome changes, 244

$-B$. japonicum: on legume nodules, type III secretion, promoter, 1087; relation to viruses, promoters, 1027; on soybean, autoregulation, 1337; on soybean, nodulation profiling, 631 ; on soybean, virusderived promoter, cover photo, August

Brassica spp.

-B. juncea, Albugo candida, compatibility with Arabidopsis, 745

-B. napus, Leptosphaeria maculans, apoptosis and leaf lesion formation, 1143

Brazelton, J. N., 1349

Brechenmacher, I., 631

Brentani, H. P., 891

Brisset, M. N., 1076

Brito, B., 597

Bronstein, P. A., 490

Bruce, C., 433

Brugidou, C., 1215

Bryant, M. K., 188

Bulgakov, V. P., 1561

Cacao, witches' broom, biotrophic and saprotrophic mycelia, 891

Cakir, C., 433

Calla, B., 631

Camehl, I., 1371

Candresse, T., 198, 1154

Cannon, S. B., 61

Capela, D., 1232

Capstick, D. S., 979

Carazzolle, M. F., 891

Carpenter, S. C. D., 1387

Carraro, D. M., 891

Cartieaux, F., 244

Cascardo, J. M., 891

Cassava vein mosaic virus

—on soybean root nodules, promoter, cover photo, August

—on soybean roots, promoter, 1027

Castanea dentata, blight, laccase gene, virulence, 1582

Cauliflower mosaic virus, on soybean root nodules: 
- promoter, cover photo, August

-relation to bacteria, 1027

Cazaux, M., 61

Celis, C., 909

Cevallos, M. A., 958

Cha, B.-J., 1582

Chalupowicz, L., 1094

Chandra-Shekara, A. C., 1316

Chao, N.-X., 411

Chardon, F., 61

Charkowski, A. O., 133, 304, 1431, 1549

Charles, T. C., 979

Chatterjee, S., 326, 1309

Che, F.-S., 1635

Chen, B., 411

Chen, K.-C., 1046

Chen, L.-F., 1036

Chen, M.-S., 70

Chen, Q., 411

Chen, R., 122

Cheng, C.-P., 1261

Chi, M.-H., 525

Chiang, C.-H., 1046

Chintamanani, S., 79

Cho, B. H., 1067, 1643

Cho, S. M., 1067, 1643

Choi, C. J., 133

Choi, J., 525

Chou, I.-C., 1261

Chu, Y.-J., 1261

Chung, G. C., 1643

Chung, H.-J., 1582

Chung, K.-R., 469

Citrus, Elsinoë fawcettii, phytotoxin, pathogenesis, conidiation, 469

Ciuffetti, L. M., 315

Clark, S. R. D., 979

Clough, S. J., 631, 1297

Cochliobolus spp.

-C. carbonum, on maize, resistance mechanisms, 79

-C. heterostrophus, on corn, MAP kinases, stress responses, 769

-C. victoriae, on Arabidopsis, victorin, LOV1 gene, 7

Coenen, C., 1349

Cohen, J. D., 1408

Collado, I. G., 1443

Colletotrichum spp.

-C. coccodes, on tomato, ammonia secretion and $\mathrm{pH}$ modulation, 1058

-C. graminicola, on corn, yeast signal sequence trap, 1325

-C. trifolii, on Medicago truncatula, resistance, 61

Collmer, A., 490, 685

Combier, J.-P., 1118

Conn, V. M., 208

Conrath, U., 1421

Constantin, G. D., 720

Contesto, C., 244

Cooper, A. J., 745, 757

Coque, L., 404

Corn (see Maize)

Cornu, A., 1471

Coronatine, Pseudomonas syringae on tomato, virulence function, 383

Cosme, A. M., 947

Costa, G. G. L., 891

Cotalano, C., 404

Courtois. B., 859

Cover photo

-Arabidopsis, soybean rust, nonhost effect, November

-Bean pod mottle virus, on soybean, gene silencing, May

-Cassava vein mosaic virus, promoter, August
—Cauliflower mosaic virus, $35 \mathrm{~S}$ promoter, August

—Epichloë festucae, $\beta$-glucuronidase expression, spikelets, February

-Figwort mosaic virus, promoter, August

- Heterodera schachtii, on Arabidopsis, December

- Phytophthora infestans, on tomato, glucanase inhibitor proteins, June

- Pseudomonas aeruginosa, computer docking analysis, September

- Pseudomonas fluorescens, on tomato seedlings, antibiotic effect, October

-Pseudomonas syringae on tomato, multiple effector proteins, April

-Rhizobium etli, on Phaseolus vulgaris, stress tolerance, July

- soybean, root nodules, profile, August

- Tobacco mosaic virus, cell-to-cell model, March

-victorin, $L O V 1$ protein, sensitivity and disease susceptibility, January

Cowling, W. A., 1143

Crucifer, black rot:

—pathogenesis process, 1036

- signal transduction systems and mutagenesis, 1128

Crute, I. R., 745

Cryphonectria parasitica, on chestnut, laccase gene, 1582

Csukai, M., 1249

Cucumber, Pseudomonas chlororaphis, galactinol signaling, 1643

Cucumber mosaic virus

—on Arabidopsis thaliana, resistance gene, 1398

—resistant transgenic plants, 20-year review, 675

da Cunha, L., 433

Daire, X., 232

Damasceno, C. M. B., 820

Danesh, D., 61

Dangl, J. L., 361, 1549

D'Antuono, A. L., 50

de Billy, F., 535

de Carvalho-Niebel, F., 535

Decroocq, V., 198

Degani, O., 769

De Giovanni, C., 30

Degrave, A., 1076

Deising, H. B., 1325

de las Nieves Peltzer, M., 1232

del Carmen Orozco, M., 958

Delgadillo, M. O., 20

Delledonne, M., 781

De Lorenzo, G., 171

Delucia, E. H., 1297

de Oliveira, B. V., 891

Desbrosses, G., 244

De Stefano, M., 781

De Vleesschauwer, D., 709

Dias, R., 891

Dicke, M., 919

Dickeya dadantii

-GacA, type III secretion, regulation, 133

- gene regulation by HrpY, hrp cluster link, 304

- on Nicotiana benthamiana, flagellar sigma factor, 1431

Dickman, M. B., 605

Dickstein, R., 404

Dmitrenok, P. S., 1561

D'Ovidio, R., 171

Dowd, S. E., 737

Droc, G., 859, 869

Dumas, B., 61

Dunning, F. M., 646

Eggenberger, A. L., 931, 937

Elbaum, M., 335
Elbaz, M., 1261

Elmore, J. M., 1027

Elsinoë fawcettii, on citrus, elsinochrome, pathogenesis and conidiation, 469

Engelberth, J., 98

Epel, B. L., 335

Epichloë festucae

- spikelets, $\beta$-glucuronidase effect on, cover photo, February

-symbiosis, lolitrem biosynthetic gene pattern, 188

Erwinia spp.

-E. amylovora: on apple, pear, and tobacco, HrpN virulence protein, 1387; on Arabidopsis thaliana, defense and cell death, proteins, 1076

-E. carotovora, quorum-sensing targets, protein and regulator, 957

-E. chrysanthemi, regulator analysis, pathogenicity, 1471

Erysiphe pisi, on Medicago truncatula, resistance, 61

Esquerré-Tugayé, M. T., 61

Fagard, M., 1076

Farmer, L., 361

Farrow, J. M., III, 1184

Favaron, F., 171

Félix, J., 518

Feng, J.-X., 411

Ferrarini, A., 781

Fester, T., 1027

Feussner, I., 98

Figwort mosaic virus

- on soybean root nodules, promoter, cover photo, August

-on soybean roots, promoter, 1027

Fischer, R., 1242

Fluhr, R., 1058

Formighiei, E. F., 891

Franche, C., 518

Franco, C. M. M., 208

Frick, M., 346

Fu, D.-Q., 564

Fudali, S., 791

Fukumoto, T., 1591

Fusarium spp.

-F. graminearum, on wheat: fusion protein, antibody and peptide, 1242; infection phases and genes, 1571

-F. oxysporum, on tomato, tomatinase, 728

$-F$. verticillioides, on maize, fumonisin, gene cluster deletion, 87

Gallou, A., 244

Gamas, P., 1118

Gao, M., 162, 294, 1184

Gao, X., 98

Garcia, J. (France), 535

García, J. A. (Spain), 20

Gardiner, D. M., 1571

Garvin, D. F., 1515

Gaubert, S., 1076

Gaudet, D. A., 346

Ge, Y.-Y., 1036

Gene regulation, Xanthomonas spp., signal

transduction system, 151

Genes

-Avr4, potato late blight, 1460

-LOV1, victorin, Cochliobolus victoriae, 7

-nirK, wheat and Azopirillum brasilense, 831

- Pi-ta, rice blast resistance gene, 396

-rolC oncogene, Agrobacterium and Rubia cordifolia, 1561

—rsmAXcc, Xanthomonas campestris on

Chinese radish, pathogenesis role, 411

-Rx, Nicotiana spp., 1154

— Vfal and Vfa2, apple scab, 448 
Gene silencing

-Allocasuarina verticillata, host transcriptional in roots, 518

—Lotus japonicus, polyubiquitin, 375

- symbiotic root nodules, virus-induced, technical advance, 720

- Tobacco mosaic virus, protein effect, 1539

- tomato to nematode, effector gene, 576

Gene transfer, lateral, fungal diseases of crop plants, review, 287

Genger, R. K., 1285

Ghabrial, S. A., 564

Gherardi, M., 535

Gherbi, H., 518

Gianinazzi-Pearson, V., 232

Giesbert., S., 808

Gilchrist, D. G., 1143

Girardin, R., 518

Girke, T., 1205

Glasner, J. D., 1549

Glazebrook, J., 1408

Gleason, C. A., 576

Glenn, A. E., 87

Globodera rostochiensis, on potato, glucanases role, 791

Göbel, C., 98

Goel, A. K., 361

Goellner, K., 1421

Goh, J., 525

Gold, S. E., 110

Golinowski, W., 791

Gomi, K., 1591

González, E. M., 622

Goodin, M. M., 1015

Gorpenchenko, T. Y., 1561

Göttfert, M., 1087

Govers, F., 433, 1460

Goverse, A., 791

Govindarajulu, M., 1027

Grant, S. R., 361, 1549

Grape

-downy mildew, glucan sulfate to induce resistance, 232

-Xylella fastidiosa, cell-to-cell signaling, 1309

Greenberg, J. T., 1285

Grenville-Briggs, L., 433

Gresshoff, P. M., 843, 1337

Grønlund, M., 720

Guenoune-Gelbart, D., 335

Gulden, S., 757

Gunn, N., 757

Guo, J., 1460

Haas, C., 988

Hadar, R., 769

Hajimorad, M. R., 931, 937

Hamada, K., 586

Hamilton, S. M., 315

Hammond-Kosack, K. E., 1193

Han, S. H., 1067

Han, S.-S., 525

Han, Z.-J., 151, 1128

Hang, X.-H., 1036

Harjunpää, H., 967

Harries, P. A., 1539

Harris, M., 70

Hase, S., 1398

Hasselmann, K. D., 1408

Haurat, J., 831

Havens, W., 564

Hayashi, M., 375

Hayashi, S., 843

He, C., 151, 555, 1128

He, G., 122

He, R., 122

He, Y.-Q., 411, 1036

He, Z., 294

Helder, J., 791
Hernández, G., 958

Herrera, C. M., 1359

Heterodera schachtii, on Arabidopsis:

-RNAs and targets, 1622; miRNAs, cover photo, December

—salicylic acid, 424

Hewezi, T., 1622

Hibi, T., 586

Higashi, S., 1175

Hill, J. H., 931, 937

Ho, F.-I., 1261

Ho, K. A., 326

Höfte, M., 709

Hohnjec, N., 622, 1118

Holub, E. B., 745, 757

Horbach, R., 1325

Horwitz, B. A., 769

Howe, P., 1622

Huang, P.-C., 1261

Huang, S.-M., 1261

Huang, T., 1242

Huesmann, C., 1443

Hugouvieux-Cotte-Pattat, N., 1471

Huguet, T., 61, 535

Hutten, R. C. B., 909

Hwangbo, H., 1643

Hyaloperonospora parasitica and Pseudomonas syringae, on Arabidopsis, resistance signaling pathways, 1285

Hypersensitivity, Turnip crinkle virus on Arabidopsis, 1316

Ichinose, Y., 1165, 1398

Igbaria, A., 769

Im, Y. J., 1643

Imperial, J., 597

Inagaki, Y., 1165

Insects, Arabidopsis, microbially induced resistance, 919

Inukai, T., 260

Ishiga, T., 383

Ishiga, Y., 383

Isogai, A., 1635

Itkin, M., 1094

Iturriaga, G., 958

Jacob, A. I., 979

Jacquet, C., 61

Jahn, C. E., 1431, 1549

Janakowski, S., 791

Janni, M., 171

Jeannotte, R., 70

Jensen, P. J., 1387

Jeon, Junhyun, 525

Jeon, J.-S., 294

Jeong, R.-D., 1316

Jia, Y., 396

Jiang, B.-L., 411, 1036

Jimenez-Teja, D., 1443

Jin, J., 424

Johal, G. S, 79

Johansen, I. E., 720

Joshi, T., 631

Joubet, J.-M., 232

Journet, E.-P., 535, 1118

Judelson, H. S., 433

Jung, H. W., 1285

Jung, M. W. M., 1460

Jurkowski, G. I., 1285

Kachroo, A., 564, 1316

Kachroo, P., 564, 1316

Kaloshian, I., 1205

Kamoun, S., 433, 820

Kang, B. R., 1067

Kang, E. Y., 1643

Kang, H.-G., 1398

Kang, S., 658
Kanyuka, K., 1193

Karczmarek, A., 791

Karplus, P. A., 315

Katagiri, F., 1408

Kawakami, S., 1398

Kema, G. H. J., 1249

Keurentjes, J. J. B., 198

Khang, C. H., 658

Kim, B. (Japan), 260

Kim, B. R. (Korea), 525

Kim, D.-H., 1582

Kim, H.-S., 1549

Kim, J.-M., 1582

Kim, K.-H., 879

Kim, K. S., 605

Kim, M. S., 737, 1643

Kim, M.-Y., 631

Kim, S., 525

Kim, Y. C., 1067, 1643

Kinkema, M., 843, 1337

Klarzynski, O., 232

Klessig, D. F., 40, 1316, 1398

Klingler, J. P., 701

Klosterman, S. J., 110

Kokkelink, L., 808, 1443

Kolmer, J A., 1515

Kolomiets, M., 98

Kopka, J., 244

Korban, S. S., 448

Koutsoudis, M. D., 1359

Krijger, J.-J., 1325

Krol, E., 1498

Krusell, L., 50

Kubota, M., 1398

Kucho, K.-i., 1175

Kurek, W., 791

Kusakabe, M., 375

Küster, H., 459, 622,1118

Kwon, B.-R., 1582

Lamattina, L., 1001

Lang, K., 1087

Langenbach, C., 1421

Lankhorst, R. K., 1249

Lara, M., 958

Laroche, A., 346

Laroche, S., 1076

Latijnhouwers, M., 433

Latunde-Dada, A.O., 745

Lee, B. N., 769

Lee, M. P., 631

Lee, S.-H., 631

Lee, Y.-H., 525, 658, 1067

Le Gall, O., 198

Lehto, H., 178

Lehto, K., 178

Lenarz-Wyatt, L., 1408

Lenman, M., 1275

Lepek, V. C., 50

Leptosphaeria maculans, on Brassica napus, apoptosis and leaf lesion formation, 1143

Lev, S., 769

Leveau, A., 1154

Levy, A., 335

Li, C., 1143

Li, H.-P., 1242

Li, M., 631, 1297

Li, Q., 294

Liang, X.-X., 411

Liao, H.-L., 469

Liao, Y.-C., 1242

Libault, M., 631

Lichocka, M., 791

Lightfoot, D. A., 507

Ligterink, W., 433

Lin, Y.-M., 1261

Lincoln, J. E., 1143

Lindeberg, M., 685 
Lindhout, P., 30

Lindow, S. E., 326, 1309

Liou, R.-F., 480

Liu, F.-L., 1046

Liu, J., 1036

Liu, L.-F., 480

Liu, Q. L., 576

Liu, Xiang, 70

Liu, Xming, 70

Loehrer, M., 1421

Lolium perenne, Epichloë festucae on, lolitrem biosynthetic gene, 188

Lommel, S. A., 879, 1015

Lorang, J. M., 7

Lotti, C., 30

Lotus japonicus

— gene silencing, polyubiquitin vectors, 375

-Mesorhizobium loti inoculation, nodule development, 50

-symbiotic and pathogenic bacteria, hemoglobin gene and nitric oxide, 1175

Loudet, O., 198

Lu, D.-K., 1261

Lu, G.-T., 411, 1036

Lu, H., 1285

Lu, Z.-X., 346

Lucas, J. A., 745

Ludwig-Müller, J., 1371

Lund, O. S., 720

Lundberg, D., 361

Lynn, J., 745

Lyons, R., 1193

Ma, B., 1549

Madsen, L. H., 1600

Maekawa, T., 375

Magnaporthe spp.

$-M$. grisea, on rice, avirulence gene family, organization and evolution, 658

$-M$. oryzae, on rice: cell wall integrity and pathogenicity, 525; protein interaction and resistance, 294; resistance gene Pi-ta, 396

Maier, T. R., 1622

Maize (see also Zea mays)

-fumonisin production and pathogenicity, 87

- leaf spot and ear mold, $\mathrm{Hm} 2$ gene, dosage, 79

—root-knot nematode, resistance genes, 98

- Southern corn leaf blight, MAP kinases, stress responses, 769

- Stewart's wilt, pathogen motility, 1359

Malnoy, M., 448

Manners, J. M., 1571

Manning, V. A., 315

Mantelin, S., 1205

Manulis-Sasson, S., 1094

Marino, D., 622

Marquez-Villavicencio, M., 1549

Martin, M. E., 490

Martin, R., 396

Martinez-Espinosa, A. D., 110

Masunaka, A., 1591

Masuta, C., 260

Matsuura, H., 260

Matthews, R., 361

May, K. J., 188

Mayetiola destructor, on wheat, carbon and nitrogen metabolism, 70

McDowell, J. M., 1285

McIntosh, M., 1498

McNellis, T. W., 1387

McSpadden Gardener, B. B., 1349

Medicago spp. (see also Alfalfa)

-M. sativa, Sinorhizobium meliloti: mutational analysis, symbiosis, 979; symbiosis and resistance, 947

- M. truncatula: anthracnose and powdery mildew resistance, genetics, 61 ; nitric oxide response genes, pathogenesis and symbiosis, 781; nodulation, regulatory genes, 1118 nodule primordium invasion, 535 ; nodules and esterase, symbiosomes, 404; symbiosis analysis, RIVET, 162

Meijer, H. J. G., 433

Meinhardt, L. W., 891

Mekuria, T., 1106

Meloidogyne spp., on tomato: gene silencing, 576; jasmonic acid signaling, 1205

-M. incognita, on maize, resistance genes, oxylipin, 98

Mendes, O., 1249

Meng, Q.-L., 411

Mercier, L., 232

Meschini, E. P., 459

Messeguer, J., 1215

Metabolism, wheat, carbon and nitrogen, Hessian fly, 70

Meyers, B. C., 646

Michelmore, R. W., 646

Min, J.-Y., 605

Mitra, R. M., 1408

Miyamoto, Y., 1591

Moënne-Loccoz, Y., 831

Mole, B., 1549

Molina-Favero, C., 1001

Mondego, J. M. C., 891

Mónica Creus, C., 1001

Moniliophthora perniciosa, biotrophic and saprotrophic mycelia, gene expression, 891

Moran, J. F., 622

Moreira, L. M., 947

Morel, J.-B., 859, 869

Morris, T. J., 879

Morroni, M., 675

Mosher, S., 40

Moya, A., 701

Multani, D. S., 79

Munkvold, K. R., 490

Munn, A. L., 1571

Murakami, E.-i., 1175

Murooka, Y., 375

Mycosphaerella graminicola, on wheat, gene discovery in plant expression, 1249

Mycotoxin (see also Toxin): fumonisin. Fusarium verticillioides on maize, 87

Myers, C. R., 685

Mysore, K. S., 383

Nagata, M., 1175

Naidu, R. A., 1015

Naito, K., 1165

Namba, S., 586

Nambiar-Veetil, M., 518

Nattkemper T. W., 219

Navarre, D. A., 564, 1205

Nelson, R. S., 1539

Neogi, P., 404

Nester, E. W., 1528

Neuhaus, H. E., 547

Newman, K. L., 326, 1309

Nicotiana spp. (see also Tobcco): potexviruses, resistance gene $R x, 1154$

$-N$. attenuata, resistance to bacteria, herbivore resistance relation, 988

-N. benthamiana: Dickeya dadantii, flagellar sigma factor, $1431 ;$ N. tabacum comparison, transgenic, viral RNA silencing suppressors, 178; plant-pathogen interaction model, review, 1015; Tobacco mosaic virus, spread by lateral diffusion, plasmodesmal desmotubule, 335

Niebel, A., 1118

Niks, R. E., 909

Nilaparvata lugens, on rice, genotype response, 122

Nodulation
- Medicago truncatula, symbiotic mutant, 535

-Mesorhizobium loti on Lotus japonicus, cylic glucan and lycopolysaccharide, 50

- pea, sucrose synthase regulation, cellular redox, 622

—regulatory genes, 1118

-Rhizobium leguminosarum, receptor kinase gene and development, 1600

-Sinorhizobium meliloti: auxotrophy and defect, 1232; on Medicago truncatula, 404

-soybean: by Bradyrhizobium japonicum, transcription profiling, 631; lipoxygenases, analysis, 843

-virus-induced gene silencing, technical advance, 720

Nottéghem, J.-L., 859, 869

Novák, O., 1371

Odinius, D., 808

Oelmüller, R., 799, 1371

Oh, C.-S., 1387

Ohtani, K., 1591

Oidium neolycopersici, on tomato, broadspectrum resistance, Mlo function, 30

Oliver, R. P., 287

O’Neill, B. F., 1297

Ott, T., 50

Ovchinnikova, E., 1600

Ozone, Soybean mosaic virus, interaction, 1297

Palacious, J. M., 597

Palanichelvam, K., 1539

Palva, E. T., 967

Panstruga, R., 30

Pantoea spp.

-P. agglomerans, gall development, quorumsensing system, 1094

-P. stewartii, on maize, surface motility factor, 1359

Papaya ringspot virus, amino acid and host specificity, 1046

Paré, P. W., 737

Pareja-Jaime, Y., 728

Park, Jong-Kun, 1582

Park, Jongsun, 525

Park, Ju-Young, 1067

Park, S,-M., 1582

Park, S.-Y., 525, 658

Park, T.-H., 909

Parker, J., 40

Patrit, O., 1076

Pavan, S., 30

Payton, M., 1106

Pea

-nodules, sucrose synthase regulation, cellular redox, 622

—Sym 37 receptor kinase gene, nodule development, 1600

Pectobacterium spp., pan-genome, nichespecificity, 1549

-P. atrosepticum, citrate uptake, virulence, 547

Peever, T. L., 1591

Peñas, G., 1215

Peng, Q., 133

Pereira, G. A. G., 891

Perino, C., 1076

Perna, N. T., 1549

Pesci, E. C., 1184

Pfeufer, E. E., 1349

Phakopsora pachyrhizi, on Arabidopsis:

-nonhost reaction, cover photo, November

-nonhost resistance, 1421

Phaseolus vulgaris

-nodulation, subtractive hybridization, 459

-Rhizobium etli, drought tolerance and seed yield, 958

Phillips, D. A., 1184 
Phosphoprotein, stress, elicitor-treated Arabidopsis, 1275

Phytophthora spp., glucanase inhibitor protein family evolution, 820

-P. infestans: on potato, avirulence gene Avr4, 1460 ; on potato and tomato, gene expression and transcriptome, 433; on Solanum microdontum, potato chromosome 4 map, 909; on tomato, glucanase inhibitor proteins, cover photo, June

$-P$. parasitica, polygalacturonases, encoding, 480

Pieterse, C. M. J., 919

Piriformospora indica, on Arabidopsis:

-auxin interaction, 1371

-drought tolerance conferred, 799

Pislariu, C., 404

Plant pathogen defense, ABA modulation, global switches, review, 709

Plasmopara viticola, on grape, resistance, priming defense responses, 232

Plum pox virus

-on Arabidopsis thaliana, quantitative trait loci, symptoms, 198

-on herbaceous and woody hosts, pathogenicity determinants, 20

Pobigaylo, N., 219

Poinsot, V., 1232

Polverari, A., 781

Potato

- cyst nematode, glucanases in host-nematode interaction, 791

- Phytophthora infestans, avirulence gene Avr4, 1460

Potato virus $X$, phloem uploading, movement proteins, regulation, 1106

Potexviruses, on Nicotiana spp., resistance gene $R x, 1154$

Pothier, J. F., 831

Powers , J. G., 879

Preston, G. M., 269

Price, A., 859

Prieto, R.-I., 597

Prigent-Combaret, C., 831

Proctor, R. H., 87

Prusky, D., 1058

Pruss, G. J., 1528

Pseudomonas spp., on tobacco, rhizobacterial elicitors of resistance, 1609

-P. aeruginosa: computer docking analysis, cover photo, September; quorum-sensing receptor, riboflavin, 1184

-P. chlororaphis: on Arabidopsis, drought tolerance, 1067; on cucumber, systemic resistance, galactinol, 1643

-P. fluorescens: root pathogens protected by antibiotic, 1349; on tomato, antibiotic effect, cover photo, October

-P. syringae: on Arabidopsis, resistance signaling pathways, 1285; on Arabidopsis, terpenoid and jasmonate signaling, 1482; on Arabidopsis, transcriptional response, regulators and phytotoxin, 1408; on Arabidopsis, virulence and amino acid sequence, 1165; on Arabidopsis, virulence on water-stressed plants, type III effector, 361; on Arabidopsis thaliana

Bradyrhizobium interaction, 244; on Lotus japonicus, hemoglobin gene and nitric oxide, 1175; on Nicotiana attenuata, resistance mediation, herbivore resistance, 988; on tomato, multiple effector proteins, cover photo, April; on tomato, nutrient assimilation pathway, apoplast, 269; on tomato, pathogenicity and coronatine, 383; on tomato, type III secretion system effector, survey, 490; virulence determinants, comparative genomics, review, 685
Pucciariello, C., 781

Puccinia triticina, on wheat, resistance and multiple metabolic pathways, 1515

Puchalski, B., 346

Pugin, A., 232, 1609

Pühler, A., 459

Puntarulo, S., 1001

Puppo, A., 781

Qi, D., 613

Qian, W., 151, 1128

Qu, F., 879

Qu, N., 294

Quilis, J., 1215

Radutoiu, S., 1600

Raja, J. A. J., 1046

Rajamani, S., 1184

Ralstonia solanacearum

-on Lotus japonicus, hemoglobin gene and nitric oxide, 1175

-on tomato and Arabidopsis, transposon mutagenesis, 1261

Ramírez, M., 958

Rayapuram, C., 988

Reedy, R. M., 133

Reese, J. C., 70

Regulator genes, Dickeya dadantii, HrpY link, 304

Reinstädler, A., 30

Renou, J.-P., 244

Resistance

-apple to scab, genes Vfal and Vfa2, 448

-Arabidopsis: defense activation, 40; to soybean rust, 1421; to Turnip crinkle virus, hypersensitivity, 1316; to white rust, protein, 757

-Arabidopsis thaliana to Cucumber mosaic virus, gene $R C Y 1,1398$

-barley to Soilborne cereal mosaic and Soilborne wheat mosaic viruses, 1193

-maize to Cochliobolus carbonum, mechanisms, 79

- Medicago truncatula, anthracnose and powdery mildew, genetic dissection, 61

-rice to blight and blast, protein interactions, 294

—rice blast, meta-analysis, review, 859

- soybean to multiple pathogens, defense signaling, oleic acid pathway, 564

- tomato to: powdery mildew, Mlo function, 30; root-knot, jasmonic acid signaling, 1205

-wheat to: Bipolaris sorokiniana, bean PGIP role, 171; leaf rust, multiple metabolic pathways, 1515

Reverchon, S., 1471

Revers, F., 198

Reviewers, acknowledgment, 3

Reymond, P., 919

Rhizobacteria, on Arabidiopsis, resistance induced to insects, 919

Rhizobium spp.

$-R$. etli: on bean, host genes and nodulation preference, 459; on Phaseolus vulgaris, drought and yield effect, 958; on

Phaseolus vulgaris, stress tolerance, cover photo, July

- R. leguminosarum: host-dependent expression in nodules, 597; receptor kinase gene, nodule development, 1600

Rho, H.-S., 525

Ricciardi, L., 30

Rice

-Acidovorax avenae, flagellin perception, flg22 receptor, 1635

-blast: cell wall integrity and pathogenicity, kinase, 525; resistance, meta-analysis, review, 859; resistance gene $P i$-ta, 396

-brown planthopper response, genotypes, 122

- defense responses, hypersensitivity, 1215

- disease regulation, ARCHIPELAGO, review, 869

-Magnaporthe grisea, AVR-Pita avirlence gene family, organization and evolution, 658

-resistance to blight and blast, OsRAR1 and OsSGT1 interaction, 294

Rico, A., 269

Riley, R. T., 87

Rimmer, S. R., 757

Rincones, J., 891

Ripoll, D. R., 820

Rissman, A. I., 1549

Ritter, C., 1371

RNA silencing

-identification assay, virus complementation, technical advance, 879

-Nicotiana spp., transgenic, suppressors, 178

Roberts, S., 433

Robinson, J. B., 1184

Roncero, M. I. G., 728

Rong, W., 555

Roosien, J., 791

Roques, N., 1232

Rose, J. K. C., 820

Rose, M. S., 769

Rostás, M., 1482

Rubia cordifolia, Agrobacterium rhizogenes, rolC oncogene, stress tolerance, 1561

Ruess, H., 79

Ruiz-Argüeso, T., 597

Ruiz-Roldán, M. C., 728

Rychagova, T., 1600

Ryu, C.-M., 1067, 1643

Sabha, M., 891

Sacher, A., 1094

Sáenz, P., 20

Sagi, G., 335

Sakurai, S., 586

Salvador, B., 20

Samac, D. A., 61

Sandbrink, H., 1249

San Segundo, B., 1215

Santos, M. R., 947

Santos, P. M., 947

Sarmiento, C., 178

Sassi, G., 40

Satellite panicum mosaic virus, capsid protein, activities, 613

Sato, M., 1408

Sato, S., 375

Sayre, R. T., 1184

Scarpari, L. M., 891

Schaffrath, U., 1421

Schneider, D. J., 685

Schober, G., 1087

Scholthof, K.-B. G., 613

Schumacher, J., 1443

Schweizer, P., 1325

Sclerotinia sclerotiorum, programmed cell death, oxalic acid as elicitor, 605

Scott, B., 188

Seay, J. R., 110

Segmüller, S., 808

Sekine, K.-T., 1398

Sella, L., 171

Serine threonine kinases, signaling and plant defense roles, review, 507

Sessa, G., 1094

Shah, J., 1398

Shahollari, B., 1371

Sharypova, L. A., 947

Shen, H.-L., 1261

Sherameti, I., 799 
Sherman, A., 1058

Sherrier, D. J., 404

Shi, H., 737

Shi, R.-P., 1242

Shimizu, T., 586

Shimoda, Y., 375, 1175

Shimoda-Sasakura, F., 1175

Shiraishi, T., 1165

Shkryl, Y. N., 1561

Sicard, O., 198

Siddiqui, S. A., 178

Simon, J.-C., 701

Simón-Mateo, C., 20

Simontacchi, M., 1001

Sinn, J. P., 1387

Sinorhizobium meliloti

-auxotrophy, nodulation defect, 1232

- gene expression analysis, RIVET, 162

-on Medicago sativa, protein secretion, resistance, and symbiosis, 947

—on Medicago truncatula: nitric oxide and symbiosis, 781; nodules and esterase symbiosomes, 404

-mutational analysis, symbiosis and catabolic diversity, 979

—regulator MucR, exopolysaccaride synthesis, 1498

-symbiosis and competitiveness genes, signature-tagged mutants, 219

Sit, T. L., 879

Sjöblom, S., 967

Sobczak, M., 791

Solanum microdontum, Phytophthora infestans, resistance, chromosome 4 map, 909

Solomon, P. S., 287

Sörensson, C., 1275

Soybean

—nodulation, Bradyrhizobium japonicum, profiling, 631

—nodulation autoregulation, downstream signals, 1337

—nodule development, lipoxygenases, molecular development, 843

- pathogens, defense signaling and resistance, 564

-roots and nodules, viral promoters, technical advance, 1027

Soybean mosaic virus

—avirulent chimeras, mutations mediated, 937

-ozone interaction, 1297

- virulence gain, mutations, 931

Spence, T., 979

Stacey, G., 631

Starr, J., 98

Stephens, A. E., 1571

Stougaard, J., 720, 1600

Strawberry vein banding virus, on soybean

roots, promoter, 1027

Strnad, M., 1371

Stuart, J. J., 70

Sturbois, B., 1154

Suárez, R., 958

Sun, J.-Y., 346

Sun, Y., 737

Suzuki, A., 1175

Suzuki, M., 586

Suzuki, T., 1165

Sweat, T. A., 7, 1349

Symbiosis, Sinorhizobium meliloti, gene expression analysis, RIVET, 162

Szymczak, S., 219

Tabata, S., 375

Taconnat, L., 244

Tagu, D., 701

Taguchi, F., 1165

Tai, C.-H., 1046

Takahashi, H., 260, 1398
Takai, R., 1635

Takayama, S., 1635

Tan, M. Y. A., 909

Tan, X., 646

Tang, D.-J., 411, 1036

Tang, J.-L., 411, 1036

Tangerine, Alternaria brown spot, toxin biosynthesis gene, RNA silencing, 1591

Tao, J., 1128

Taylor, C. G., 1027

Teillet, A., 535

Tepfer, M., 675

Teplitski, M., 162, 1184

Tharreau, D., 859, 869

Theres, K., 30

Thompson, J. R., 675

Thurber, C. S., 433

Tian, M., 40

Tikhonovich, I., 1600

Tobacco (see also Nicotiana spp.)

-fire blight, nonhost effect, harpin protein, 1387

-Pseudomonas spp., systemic resistance, rhizobacterial elicitors, 1609

Tobacco mosaic virus

—cell-to-cell spread, model, cover photo, March

-on Nicotiana benthamiana, lateral diffusion in plasmodesmal desmotubule, 335

-Nicotiana tabacum, proteins, dosage, gene silencing, 1539

-on tobacco, cell-to-cell movement, novel protein, 586

Toffanin, A., 597

Tomatinase, Fusarium oxysporum, tomato, 728

Tomato

-Azospirillum brasilense on, nitric oxide effect on roots, 1001

-bacterial speck, coronatine function, 383

-Colletotrichum on fruit, ammonia and $\mathrm{pH}$ relation, 1058

-Fusarium wilt, tomatinase and virulence, 728

—powdery mildew, Mlo function loss, 30

- Pseudomonas syringae: assimilation pathway, apoplast, 269; type III secretion system effector, 490

-Ralstonia solanacearum, transposon mutagenesis, 1261

-root-knot nematode, jasmonic acid signaling, 1205

Tomlinson, J., 98

Tör, M., 757

Torres, M. A., 361

Touraine, B., 244

Toxin (see also Mycotoxin)

-ACT, Alternaria alternata on tangerine, RNA silencing, 1591

- coronatine, Pseudomonas syringae on Arabidopsis, 1408

- Ptr ToxA, wheat, internalization requirement, 315

Toyoda, K., 1165

Tripathi, S., 799

Trouvelot, S., 232

Truve, E., 178

Tsuge, T., 1591

Tudzynski, B., 1443

Tudzynski, P., 808, 1443

Turnip crinkle virus, on Arabidopsis:

-resistance and hypersensitivity, 1316

—veinal necrosis and HR-like cell death, 260

Uchimi, T., 1175

Udvardi, M., 50

Ugalde, R. A., 50

Uppalapati, S. R., 383

Urbanczyk-Wochniak, E., 383
Urbany, C., 547

Ustilago maydis, pheromone-responsive mating and morphogenesis, 110

Vadassery, J., 1371

Vaissayre, V., 518

Valent, B., 658

Vance, V., 1528

van der Burgt, A., 1249

van der Heijt, W. H. W., 1609

van der Lee, T. A. J., 1249

van de Vondervoort, P. J. I., 1460

van Eck, H. J., 909

Van Gijsegem, F., 1471

van Kan, J., 808

van Loon, L. C., 919, 1609

Van Oosten, V. R., 919

Van Pelt, J. A., 919

van Poppel, P. M. J. A., 1460

van West, P., 433

Varma, A., 799, 137

Varnier, A.-L., 232

Venturia inaequalis, on apple, receptor-like genes and resistance, 448

Venus, Y., 1371

Verchot-Lubicz, J., 1106

Veremeichik, G. N., 1561

Vergne, E., 869

Verstappen, E. C. P., 1249

Victorin, $L O V 1$ protein, disease susceptibility, cover photo, January

Vilas-Boas, L. A., 891

Visser, R. G. F., 30, 909

Vodkin, L. O., 631

von Bodman, S. B., 1359

Voroshilova, V., 50

Waalwijk, C., 1249

Walker, A. R., 208

Wan, J., 646

Wang, Jianjun, 294

Wang, Jaw-Fen, 1261

Wang, L., 294, 555, 1408

Wang, X., 122, 1359

Wang, Y., 122, 294

Wangdi, T., 383

Wei, K., 411

Wendehenne, D., 1609

Wenzel, M., 1087

Wheat

- crown rot, infection phases and genes, 1571

-Fusarium head blight, resistance antibody and peptide, 1242

- growth-promoting rhizobacterium, nitrite reductase gene, 831

- Hessian fly, carbon and nitrogen metabolism, 70

-leaf rust, resistance and multiple metabolic pathways, 1515

- lipid transfer proteins, antifungal properties, 346

-Ptr ToxA requirement for internalization, 315

- Septoria tritici blotch, gene discovery and plant expression, 1249

-transgenic, resistance to Bipolaris sorokiniana, bean PGIP role, 171

Whisson, S. C., 433, 1460

White, R. G., 1571

Williams, L. D., 87

Williamson, V. M., 576

Willis, D. K., 1431

Wilson, K. A., 404

Win, J., 820

Windass, J., 433

Wirsel, S. G. R., 1325

Wisniewski-Dyé, F., 831

Wlodarczyk, A., 1471 
Wolpert, T. J., 7

Wong, A., 958

Wood, A. J., 507

Woods-Tör, A., 745

Wu, C.-H., 480

$\mathrm{Wu}, \mathrm{J} ., 988$

Wubben, M. J. E., 424

Xanthomonas spp., signal transduction system, genomics, 15

$-X$. campestris: on Chinese radish, gene rsmAXcc, pathogenesis, 411; on crucifers, signal transduction systems, mutagenesis, 1128; pathogenesis process, DsbB requirement, 1036; type III secretion regulators, virulence, 555

$-X$. oryzae, on rice, resistance, protein interaction, 294

Xie, Q.-G., 1205

$\mathrm{Xu}, \mathrm{D} ., 631$

Xu, M., 448
Xu, W. W., 1515

Xylella fastidiosa, on grape, cell-to-cell signaling, 1309

Yamaji, Y., 586

Yamamoto, M., 1591

Yan, H.-Z., 480

Yang, C.-H., 133, 304, 1549

Yang, K. Y., 1067, 1643

Yang, M.-S., 1582

Yang, S., 133

Yap, M.-N., 304

Yeast signal sequence trap, Colletotrichum graminicola on corn, 1325

Yeh, S,-D., 1046

Yi, X., 133

Yoo, S., 525

Yoshida, A., 586

Yoshii, A., 586

Young, N. D., 61

Yuan, H., 122
Zaitlin, D., 1015

Zanetti, M. E., 459

Zappel, N. F., 30

Zea mays (see also Maize): Colletotrichum graminicola, yeast signal sequence trap, 1325 Zehner, S., 1087

Zeier, J., 1482

Zhang, H., 737

Zhang, J.-B., 1242

Zhang, Q., 133

Zhang, X., 188

Zhang, Y., 294

Zheng, Z., 30

Zhong, C., 518

Zhou, F., 40

Zhu, L., 70, 122

Zhukov, V., 1600

Zhuravlev, Y. N., 1561

Zimeri, A. M., 87

Zitomer, N. C., 87

Zwiers, L.-H., 1249 